\title{
Exciplex Formation and Electron Transfer in a Vinyl Copolymer
}

\author{
Kaoru IwaI, * Masaoki Furue, ${ }^{*}$ Shun-ichi NozaKura, ${ }^{*}$ \\ Yasuhiko SHIROTA, ${ }^{* *}$ and Hiroshi MIKAWA** \\ *Department of Polymer Science, Faculty of Science, Osaka University, \\ Toyonaka, Osaka 560, Japan. \\ **Department of Applied Chemistry, Faculty of Engineering, \\ Osaka University, Suita, Osaka 565, Japan.
}

(Received July 25, 1979)

\begin{abstract}
Studies have been made on the exciplex formation in a vinyl copolymer, its characteristics, and the electron-transfer process in polar solvents using a $p$ - $N, N$-dimethylaminostyrene- $p$-cyanostyrene copolymer. The copolymer was found to exhibit a much more intense intramolecular exciplex fluorescence than the model $N, N$-dimethylaniline-cyanobenzene system where the intermolecular exciplex formation occurs. The copolymer system has an advantage over the model system in the exciplex formation process but suffers a disadvantage in the process of ionic dissociation in polar solvents. The ionic dissociation for the copolymer system, however, was attained in the presence of a stronger electron acceptor, $p$-dicyanobenzene, in polar solvents. The results of flash photolysis and exciplex quenching indicated that the $p$-dicyanobenzene anion radical was generated by successive electron transfer from the cyanobenzene anion radical portion in the copolymer to $p$-dicyanobenzene. That is, in the copolymer system, the interaction between the pendant donor and acceptor groups is favored even in the presence of a stronger electron acceptor.

KEY WORDS Exciplex / Intramolecular Exciplex / $p$ - $N, N$-dimethylaminostyrene / $p$-Cyanostyrene / Copolymer / Ion Radicals / Exciplex Quenching / Flash Photolysis / Electron Transfer / Charge Separation /
\end{abstract}

An essential primary step in the conversion of light energy into chemical potential is the photoionic disssociation of an electron donor-acceptor system. In order to study a possible contribution of polymeric systems to this conversion process, we have investigated the photophysical and photochemical behaviors of polymers containing both electron donor and acceptor groups.

Though numerous studies have been made of the intramolecular excimer formation in vinyl polymers ${ }^{1,2}$ with pendant aromatic groups such as poly(styrene) ${ }^{3} \quad \operatorname{poly}(N$-vinylcarbazole $),{ }^{4} \operatorname{poly}(2-$ vinylnaphthalene $)^{5}$ etc., no studies have been made of the intramolecular exciplex formation in vinyl polymers except for some studies on condensation polymers $^{6-10}$ containing electron donor and acceptor moieties.

We have prepared vinyl copolymers with $\mathrm{N}, \mathrm{N}$ - dimethylaniline and cyanobenzene moieties as pendant groups by copolymerizing $p$ - $N, N$-dimethylaminostyrene and $p$-cyanostyrene. It is the purpose of this paper to report the intramolecular exciplex formation in the copolymer, its characteristics, and the process of electron transfer in comparison with the low-molecular-weight model system, $N, N$-dimethylaniline-cyanobenzene system where the intermolecular exciplex formation occurs. The interaction between electronically excited $N, N$ diethylaniline with ground-state cyanobenzene has been briefly reported by Beens and Weller. ${ }^{11}$

The abbreviations used in this paper are as follows: DMAS, $p-N, N$-dimethylaminostyrene; CS, $p$-cyanostyrene; DMA, $N, N$-dimethylaniline; CB, cyanobenzene; DCB, $p$-dicyanobenzene. 


\section{EXPERIMENTAL}

\section{Monomers}

DMAS was prepared from $p$ - $N, N$-dimethylaminobenzaldehyde and methylmagnesium bromide according to the method of Walling. ${ }^{12}$ bp $338-$ $348 \mathrm{~K}(0.1 \mathrm{mmHg})\left[\right.$ lit. $^{12}$ bp $\left.413 \mathrm{~K}(1 \mathrm{mmHg})\right]$.

CS was prepared in three steps from $p$ aminoacetophenone according to the following scheme.

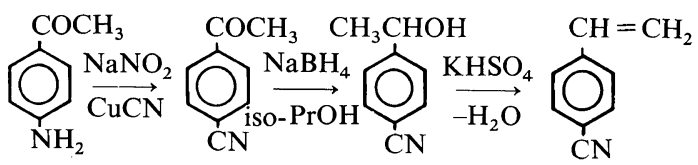

$p$-Cyanoacetophenone was obtained from $p$ aminoacetophenone according to the method of Suzuki. ${ }^{13}$ Yield, $78 \%$; bp $371 \mathrm{~K}(3 \mathrm{mmHg})$; mp $330-331.5 \mathrm{~K}$. [lit. ${ }^{13} 70-78 \%$ yield; mp 333$334 \mathrm{~K}$; bp $411-413 \mathrm{~K}(12 \mathrm{mmHg})]$.

A solution of $p$-cyanoacetophenone $(72 \mathrm{~g})$ in 2-propanol $(200 \mathrm{ml})$ was placed in a $500 \mathrm{ml}$ roundbottomed flask provided with a mechanical stirrer and a dropping funnel. A solution of $\mathrm{NaBH}_{4}(10 \mathrm{~g})$ in 2-propanol $(250 \mathrm{ml})$ was added dropwise in a period of about thirty minutes at room temperature. Following this addition, the reaction mixture was warmed up to $323 \mathrm{~K}$ and maintained at this temperature for 6 hours. The reaction mixture was treated with aq $\mathrm{NaOH}(10 \%, 100 \mathrm{ml})$ and then acidified with aq acetic acid $(50 \mathrm{vol} \%)$. After the concentration of this solution in vacuum, the organic layer was extracted with ether $(100 \mathrm{ml} \times 3)$, washed with water $(50 \mathrm{ml} \times 3)$, dried over $\mathrm{MgSO}_{4}$, and distilled under reduced pressure to give 1- $(p-$ cyanophenyl)ethanol. The yield was $60 \mathrm{~g}(82.6 \%)$; bp $391.2-393.5 \mathrm{~K}(1 \mathrm{mmHg})$. [lit. ${ }^{14} 88 \%$ yield by the Meerwein-Ponndorf reduction; bp $428-431 \mathrm{~K}$ $(6 \mathrm{mmHg})]$.

CS was prepared by the dehydration of the 1-( $p$ cyanophenyl)ethanol over $\mathrm{KHSO}_{4}{ }^{15}$. Yield, $27 \%$; bp $323-326 \mathrm{~K}$ ( $2 \mathrm{mmHg}$ ). [lit. ${ }^{15}$ yield, $29 \%$; bp $375-377 \mathrm{~K}(12 \mathrm{mmHg})]$.

\section{Polymers}

Homopolymerization was carried out in a DMF solution and initiated by AIBN at $333 \mathrm{~K}$ after degassing the solution by four freeze-pump-thaw cycles on a vacuum line. Copolymerization of DMAS and CS was carried out in the same way as the homopolymerization. The polymers were isolated by precipitation with methanol and purified by at least two or three dissolution (DMF)precipitation (methanol) steps followed by drying under reduced pressure. The polymers used in this paper are listed in Table I.

\section{Other Materials}

DMA was purified from a commercial product by fractional distillation. $\mathrm{CB}$ was purified from a commercial product by fractional distillation after drying over $\mathrm{P}_{2} \mathrm{O}_{5}$. DCB was sublimed under reduced pressure and recrystallized from acetone. Anhydrous DMF was obtained by removing water as benzene azeotrope, followed by fractional distillation through a Widmer column. DMSO was fractionally distilled through a Widmer column under reduced pressure. THF and 2-methyltetrahydrofuran (2-MeTHF) were left standing overnight on $\mathrm{CaCl}_{2}$, refluxed over $\mathrm{LiAlH}_{4}$ or sodium and then fractionally distilled through a Widmer column.

\section{Measurement}

Fluorescence spectra were recorded by means of a UNION FS-401 spectrofluorometer with a thermostatically controlled cell compartment. Dilute DMAS-CS copolymer solutions and their model systems were made free from oxygen by freezepump-thaw cycles on a vacuum line or by purging with $\mathrm{N}_{2}$ gas. The concentration of the DMA unit was about $5 \times 10^{-5} \mathrm{moll}^{-1}$ unless otherwise indicated.

Absorption spectra were measured with a Hitach1 model 124 spectrophotometer.

Transient absorption spectra were obtained photographically with Fuji Neopan SSS films. The apparatus for flash spectroscopy is almost the same as that described by Yamamotc et al. ${ }^{16}$ The concentration of DMA groups was about $4 \times 10^{-4}$

Table I. Radical polymerization of vinyl monomers

\begin{tabular}{lrcc}
\hline & Time $/ \mathrm{h}$ & Yield/\% & Mol wt $^{\mathrm{a}}$ \\
\hline PDMAS & 3 & 9.1 & 10000 \\
P(DMAS-co-CS) & 20 & 41.5 & 40000 \\
PCS & 2 & 35.7 & - \\
\hline a & \\
\multicolumn{4}{l}{ Measured by GPC. } \\
[AIBN] $=5 \times 10^{-3}$ moll $^{-1}$; [DMAS] $+[\mathrm{CS}]=1 \mathrm{moll}^{-1}$; \\
solvent, DMF; polymerization temperature, $60^{\circ} \mathrm{C}$.
\end{tabular}

Polymer J., Vol. 12, No. 2, 1980 
moll $1^{-1}$. A freshly prepared solution was degassed by several freeze-pump-thaw cycles, and then sealed into a cylindrical silica cell, $1.5 \mathrm{~cm}$ in diameter and $20 \mathrm{~cm}$ in length. An aqueous solution of $(5 \%)$ potassium monoterephthalate was used as a filter solution with a thickness of about $5 \mathrm{~mm}$, so as to cut off any light of wavelengths below $305 \mathrm{~nm}$.

The lifetime measurement was made by the pulse method with a $\mathrm{N}_{2}$ gas laser. Solution samples having an adequate concentration (absorbance of $337 \mathrm{~nm}$ was about 0.8 ) of DMA groups in dioxane or other solvents were sealed in $3 \mathrm{~mm}$ Pyrex glass capillary tubes after several freeze-pump-thaw cycles.

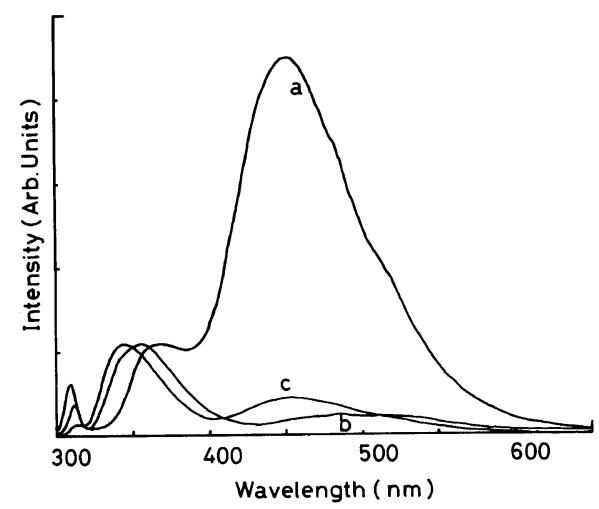

Figure 1. Fluorescence spectra in dioxane solution at $25^{\circ} \mathrm{C}$ : (a) P(DMAS-co-CS); (b) PDMAS-CB system; (c) DMA-CB system; [DMA unit], $5 \times 10^{-5} \mathrm{moll}^{-1}$; [CB unit], $3 \times 10^{-5} \mathrm{moll}^{-1}$; [DMA], $5 \times 10^{-5} \mathrm{moll}^{-1}$; [CB], $5 \times 10^{-2} \mathrm{moll}^{-1}$.

\section{RESULTS AND DISCUSSION}

\section{Characteristics of Intramolecular Exciplex in DMAS-CS Copolymer}

In Figure 1 are shown the emission spectra of the DMAS-CS copolymer and of the model systems, $\operatorname{poly}(p-N, N$-dimethylaminostyrene)(PDMAS)-CB and DMA-CB, obtained by the selective excitation of DMA (or DMA unit). The copolymer was found to exhibit an intense, broad exciplex fluorescence at ca. $450 \mathrm{~nm}$ together with a monomer fluorescence of the DMA unit at $360 \mathrm{~nm}$ in dioxane at $298 \mathrm{~K}$. Since such a strong exciplex fluorescence was observed for the copolymer in a dilute solution $\left(\mathrm{ca} .5 \times 10^{-5} \mathrm{~mol}\right.$ $1^{-1}$ ) where the inter-polymer interaction is negligibly small as is evident for concentration dependency of the $I_{\mathrm{E}} / I_{\mathrm{M}}$ in Figure 2, the exciplex formed in the copolymer is considered to be of the intramolecular

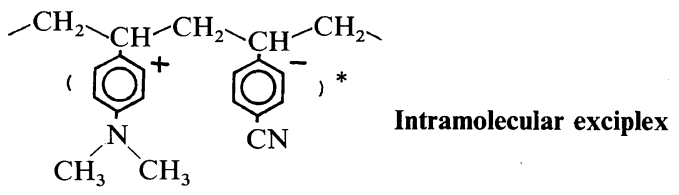

nature. The lifetime and band position of the exciplex fluorescence for the copolymer and the model systems are listed in Table II. Irrespective of the inter- or intra-molecular nature of the exciplex, the copolymer system and the DMA-CB system showed similar $\tau_{\mathrm{E}}$ and $\lambda_{\max }$ values with each other; however, it is noteworthy that the PDMAS-CB system differed greatly from the above two systems particularly in the values of their fluorescence

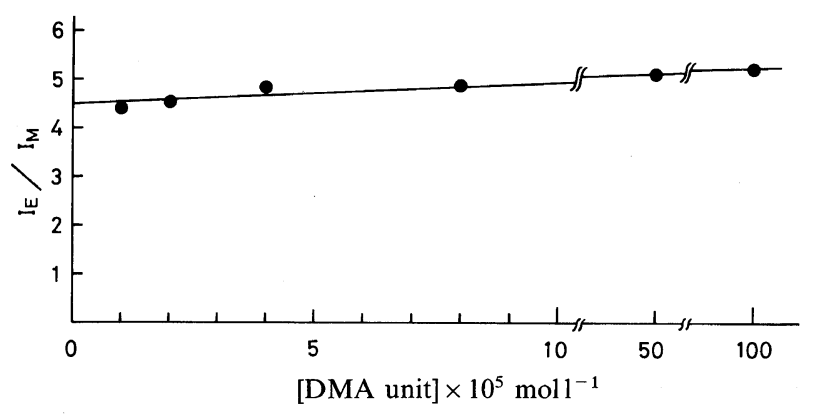

Figure 2. Dependence of the relative intensity $\left(I_{\mathrm{E}} / I_{\mathrm{M}}\right)$ of the exciplex fluorescence to the DMA monomer fluorescence for the copolymer on the concentration of DMA unit in dioxane solution. 
Table II. Lifetime and band position of exciplex fluorescence

\begin{tabular}{crc}
\hline \multicolumn{1}{c}{ System } & $\tau_{\mathrm{E}} / \mathrm{ns}$ & $\lambda_{\text {max }} / \mathrm{nm}$ \\
\hline P(DMAS-co-CS) & 99 & 450 \\
DMA $^{\mathrm{a}} \mathrm{CB}^{\mathrm{b}}$ & 140 & 454 \\
${\text { PDMAS }+\mathrm{CB}^{\mathrm{b}}}$ & 27 & 480,520 \\
\hline
\end{tabular}

a Intramolecular exciplex.

b Intermolecular exciplex.

lifetimes. In regard to the latter system, a possibility of the formation of either an exciplex, differing from the usual exciplex or a triple exciplex, would be conceivable.

The characteristic features observed for the intramolecular exciplex formed in the copolymer system are the ease of its formation and the greater intensity of its fluorescence compared with that of the intermolecular exciplex. The relative intensity of the exciplex fluorescence to the DMA monomer fluorescence $\left(I_{\mathrm{E}} / I_{\mathrm{M}}\right)$ for the copolymer ([DMA unit $]=5 \times 10^{-5} \mathrm{moll}^{-1}, \quad[\mathrm{CB}$ unit $]=$ $\left.3 \times 10^{-5} \mathrm{moll}^{-1}\right)$ is $c a .10$ times larger than that for the DMA-CB system of the comparable DMA concentration and a much larger $\mathrm{CB}$ concentration $\left([\mathrm{CB}]=5 \times 10^{-2} \mathrm{moll}^{-1}\right)$. Hence, $\mathrm{CB}$ units incorporated in the copolymer are much more effective for the formation of the exciplex than the free CB. The copolymer has a higher local concentration of electron donor and acceptor chromophores mostly separated by the trimethylene chains. Thus the intramolecular exciplex formation between adjacent pendant donor and acceptor groups can be easily attained. The ease of the intramolecular exciplex formation in the copolymer was shown also from the temperature dependence of the fluorescence spectrum of the copolymer (Figure 3). Generally the intermolecular exciplex formation does not occur at $77 \mathrm{~K}$ because of the restriction of the diffusion process. Also, in the case of the intramolecular excimer in polymer systems ${ }^{1-5}$, the ratio of the intensity of the excimer fluorescence to the monomer fluorescence at $77 \mathrm{~K}$ is much smaller as compared with that at room temperature because of the restricted molecular motion at $77 \mathrm{~K}$. In the present intramolecular exciplex system, however, exciplex fluorescence was clearly observed even at $77 \mathrm{~K}$, the band position of the exciplex fluorescence being shifted to a shorter wavelength. These facts suggest that the geometrical restriction for the exciplex formation is looser than that required of the excimer formation in polymer systems, namely, two aromatic groups should not necessarily lie face to face at a distance of $c a .3 .5 \AA$ as accepted for the sandwich excimer. It should be noted here that no significant ground-state interaction between the chromophores in the copolymer was observed in the absorption spectra which are shown in Figure 4.

Another feature characteristic of the intramolecular exciplex formed in the copolymer is that the exciplex fluorescence is less sensitive to solvent polarity than the intermolecular exciplex fluorescence of the low-molecular-weight model system. Generally, it has been accepted that the band position of the intermolecular exciplex fluorescence

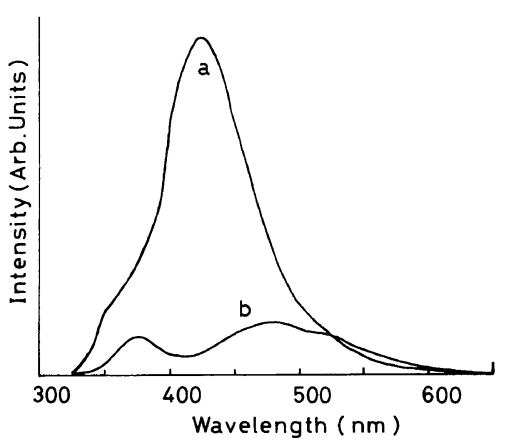

Figure 3. Fluorescence spectrum of $\mathrm{P}(\mathrm{DMAS}-\mathrm{co}$-CS) in 2-MeTHF-DMF $(80: 20)$ solution at (a) liquid $\mathrm{N}_{2}$ temperature and (b) room temperature.
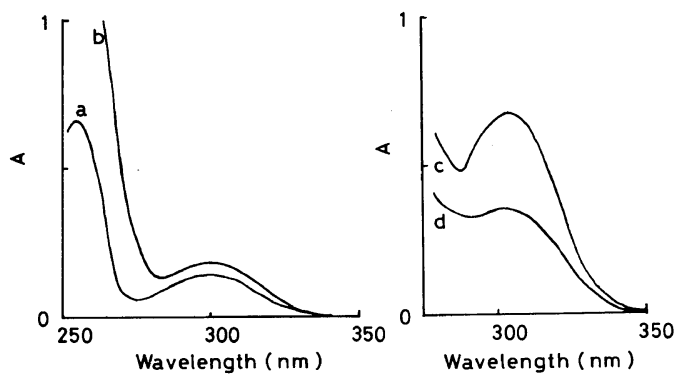

Figure 4. Absorption spectra: (a) DMA in dioxane, [DMA], $5 \times 10^{-5} \mathrm{moll}^{-1}$; (b) PDMAS in dioxane, [DMA unit], $9.5 \times 10^{-5} \mathrm{moll}^{-1}$; (c) PDMAS in DMF, [DMA unit], $3 \times 10^{-4} \mathrm{moll}^{-1}$; (d) P(DMAS-co-CS) in DMF, [DMA unit], $1.7 \times 10^{-4} \mathrm{moll}^{-1}$ and [CB unit], $1 \times 10^{-4}$ $\mathrm{moll}^{-1}$ 
undergoes a red shift and its intensity decreases as the solvent polarity increases. As compared with the case for the intermolecular exciplex fluorescence of the model system, the degree of the red shift was found to be smaller for the intramolecular exciplex fluorescence of the copolymer (Table III). This may be due to the effect of the local solvent polarity of the copolymer. Too, it should be noted that the intramolecular exciplex fluorescence of the copolymer was detected in polar solvents such as DMF or DMSO, where the intermolecular exciplex fluorescence of the model system did not appear (Figure 5). These features have been observed also for other polymeric exciplex systems. ${ }^{6,9,10}$

\section{Electron-Transfer Process}

In the preceding section, we described how the intramolecular exciplex fluorescence band of the copolymer was detected in polar solvents; however, the other important fact is that the intensity of the intramolecular exciplex fluorescence band decreases greatly as the solvent polarity increases. The results are shown in Figure 6, where the effect of the solvent polarity on the intensity of the fluorescence band was examined in a mixed solvent of dioxaneDMSO, the solvent polarity being altered by changing their composition ratio. In view of the intermolecular exciplex formation system of lowmolecular-weight compounds yielding ion radicals in polar solvents, the above result suggests that nonfluorescent ion radicals of the pendant donor and acceptor groups in the copolymer are generated in polar solvents. However, ionic dissociation of the ion radicals would be improbable since the electron donor and acceptor chromophores are covalently

Table III. Solvent effect on exciplex emissions

\begin{tabular}{lcc}
\hline \multirow{2}{*}{ Solvent } & \multicolumn{2}{c}{$\lambda_{\max } / \mathrm{nm}$} \\
\cline { 2 - 3 } & $\mathrm{DMA}+\mathrm{CB}$ & $\mathrm{P}(\mathrm{DMAS}-$-co-CS $)$ \\
\hline Benzene & 434 & 443 \\
Dioxane & 454 & 450 \\
THF & 470 & 456 \\
Dimethoxy- & 480 & 462 \\
ethane & - & 500 \\
DMF & - & 520 \\
DMSO & & \\
\hline
\end{tabular}

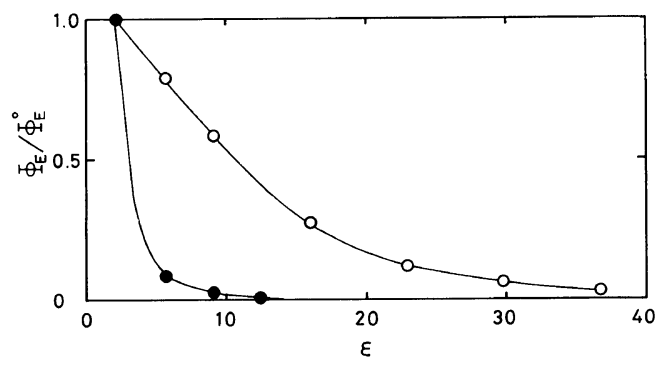

Figure 5. Dependence of relative intensity of exciplex fluorescence on slovent polarity: (O) P(DMAS-co-CS); (๑) DMA-CB system.

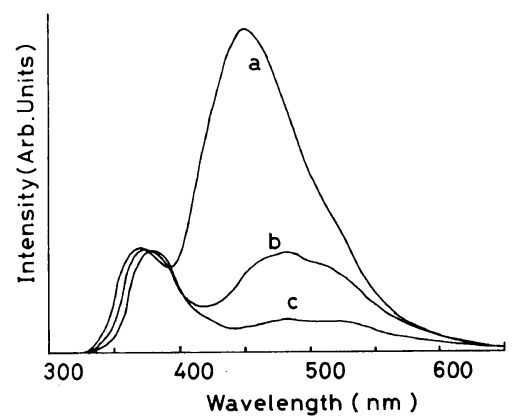

Figure 6. Fluorescence spectra of the copolymer in mixed solvent: (a) dioxane-DMSO (100:0); (b) dioxane-DMSO $(80: 20)$; (c) dioxane-DMSO $(60: 40)$.

bound to the polymer chain.

An attempt was made to demonstrate the formation of ion radicals for the copolymer in polar solvents by means of $\mu$ second flash spectroscopy. However, transient absorption of the donor cation radical and the acceptor anion radical could be obtained for neither the copolymer system nor the model DMA-CB system. The ion radicals resulting from the electron transfer, i.e., DMA cation radical and $\mathrm{CB}$ anion radical, would have very short lifetimes to be detected by the $\mu$ second flash photolysis. On the other hand, when a stronger electron acceptor, $p$-dicyanobenzene (DCB), was added to the copolymer solution, the generation of DCB anion radical was confirmed. Figure 7 shows the transient absorption spectra obtained by the selective excitation of DMA (or the DMA unit) for both the model DMA-DCB system and the copolymer-DCB system. The band with a maximum at $344 \mathrm{~nm}$ is assigned to the DCB anion radical owing to its identity with the absorption spectrum of the 

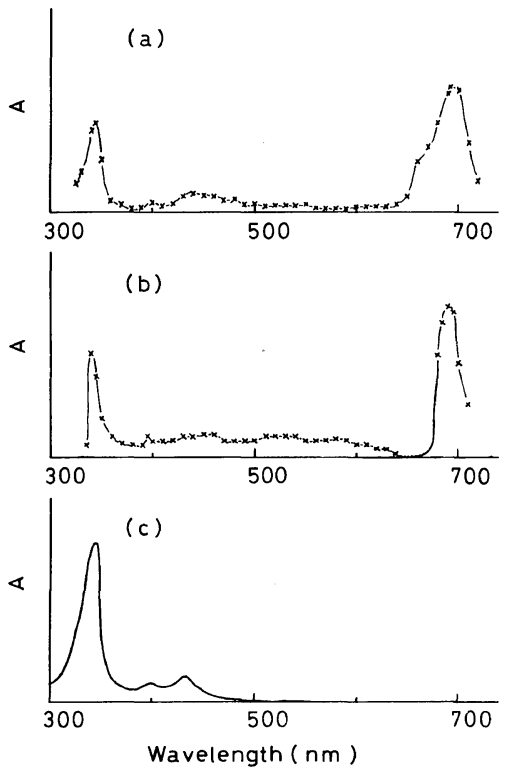

Figure 7. Transient absorption spectra of (a) DMADCB system in ethanol, $40 \mu$ s after flash, (b) P(DMAS-coCS)-DCB system in DMF, $55 \mu$ s after flash, and (c) DCB-Na in THF.

chemically prepared DCB anion radical (Figure 7(c)). The band which appeared at $690 \mathrm{~nm}$ seems to be an overtone of the above band. No absorption band assignable to the DMA cation radical $\left(\lambda_{\max } c a\right.$. $470 \mathrm{~nm}^{17}$ ) was obtained.

Two mechanisms are conceivable for the formation of the DCB anion radical. One is the successive electron-transfer from the $\mathrm{CB}$ anion radical portion formed in the copolymer to $\mathrm{DCB}$, and the other is the direct electron transfer from the excited singlet DMA (DMA*) unit to DCB. However, the result of the exciplex quenching described below indicates that the former mechanism is of primary importance. As shown in Figure 8, the intramolecular exciplex fluorescence of the copolymer was found to be sharply attenuated by DCB in non-polar solvents. Likewise, the lifetime of the exciplex fluorescence was sharply attenuated by DCB as shown in Figure 9. The rate constants obtained for the exciplex quenching were almost the same between intensity and lifetime measurements (Table IV). Since the lifetimes of the exciplex fluorescence and of the monomer DMA fluorescence differ greatly from each other, this indicates that they are not in dynamic equilibrium with each other. The fact that the lifetime of the exciplex was quenched by DCB added indicates that the exciplex quenching by DCB is not caused by the quenching of the DMA* unit by DCB but results from the interaction between the exciplex and DCB. The quenching constants and the estimated rate constants are given in Table IV. Although the monomer fluorescence of the DMA unit in the copolymer was also quenched by DCB, the quenching resulted from the interaction of free residual DMA* units with DCB. It may be concluded that the pendant DMA and CB chromophores in the copolymer effectively produce the fluorescing intramolecular exciplex in non-polar solvents and the non-fluorescent ion radicals in polar solvents, the electron transfer going from the resulting $\mathrm{CB}$ anion radical to the stronger electron acceptor DCB added.

In order to gain an insight into the process of the ion radical formation, the dependence of the lifetime $\left(\tau_{\mathrm{E}}\right)$ and quantum yield $\left(\Phi_{\mathrm{E}}\right)$ of the exciplex fluorescence on the solvent polarity was examined. As shown in Figure 10, it was observed that $\Phi_{\mathrm{E}}$ decreases more strikingly than $\tau_{\mathrm{E}}$ as the solvent polarity increases. A similar result has been reported by Weller ${ }^{18}$ for the intermolecular exciplex system of low-molecular-weight donor and acceptor compounds. They proposed the concept that ion radicals are generated by two concurrent processes, i.e., via the exciplex and directly from the encounter complex. The present result suggests that essentially the same mechanism operates also for the formation

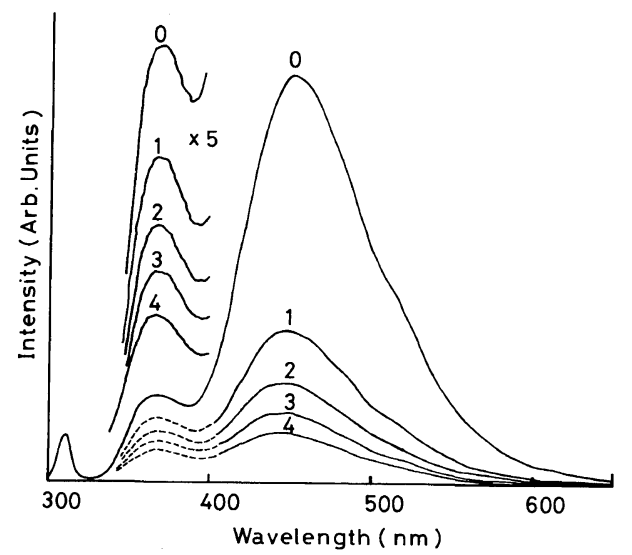

Figure 8. Fluorescence spectra of the P(DMAS-coCS)-DCB system in dioxane solution. [DCB], moll $^{-1}: 1$, $5.4 \times 10^{-3} ; 2,9.9 \times 10^{-3} ; 3,1.6 \times 10^{-2} ; 4,2.3 \times 10^{-2}$. 


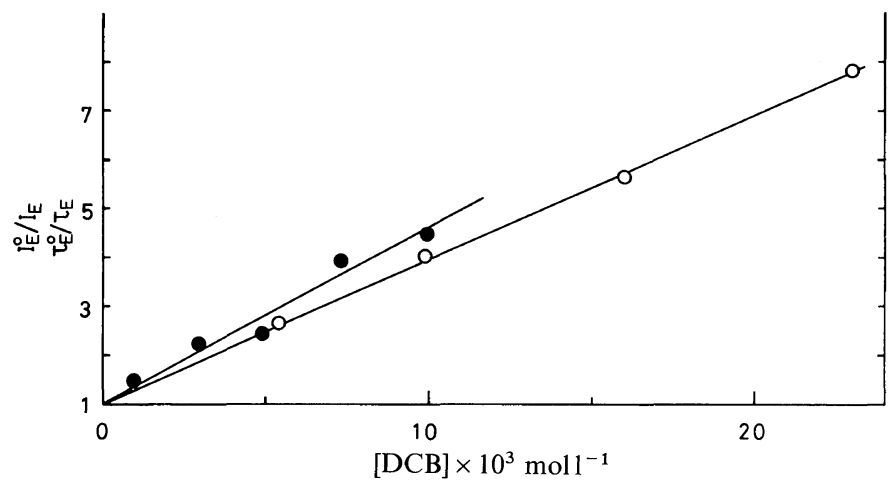

Figure 9. Stern-Volmer plots for the copolymer-DCB system in dioxane solution: $(\bigcirc) I_{\mathrm{E}}{ }^{0} / I_{\mathrm{E}} ;(\boldsymbol{O}) \tau_{\mathrm{E}}{ }^{0} / \tau_{\mathrm{E}}$.

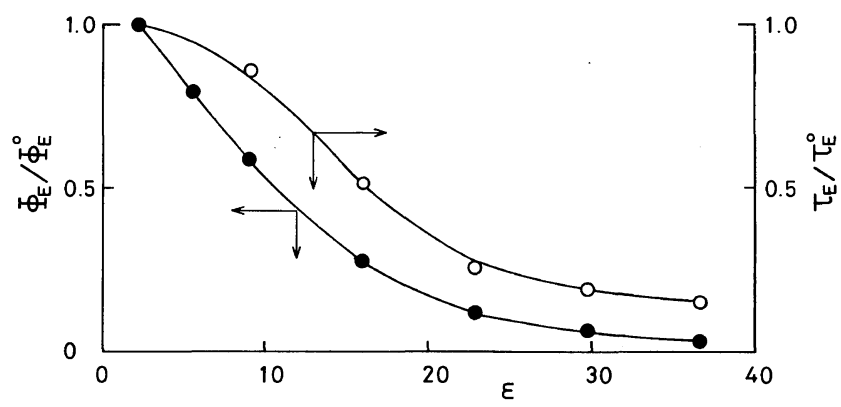

Figure 10. Dependence of relative intensity and relative lifetime of the exciplex fluorescence of the copolymer on solvent polarity: $(\bigcirc) \Phi_{\mathrm{E}} / \Phi_{\mathrm{E}}{ }^{0} ;(\bigcirc) \tau_{\mathrm{E}} / \tau_{\mathrm{E}}{ }^{0}$.

Table IV. Quenching constant ${ }^{\mathrm{b}}$ for DMA* and exciplex fluorescence by CB and DCB

\begin{tabular}{lcc}
\hline \multirow{2}{*}{ System } & \multicolumn{2}{c}{$k$ (quenching) $\times 10^{-9} / \mathrm{moll}^{-1} \mathrm{~s}^{-1}$} \\
\cline { 2 - 3 } & DMA $^{* a}$ & Exciplex \\
\hline DMA* $^{*}$ CB & $>3.1$ & - \\
PDMAS* $^{*}$ CB & $>2.9$ & - \\
DMA* $^{*}$ DCB & $>6.3$ & - \\
P(DMAS*-co-CS) +DCB & $>7.0$ & $3.03 .6^{\mathrm{c}}$ \\
\hline
\end{tabular}

a Lifetimes of DMA* and DMA* unit monomer fluorescence are smaller than $10 \mathrm{~ns}$.

b Determined by fluorescence intensity measurements.

c Determined by fluorescence lifetime measurements.

of ion radicals in the copolymer system as shown in Scheme 1. Notations $k_{\mathrm{q}}, k_{\mathrm{IP}}$ and $k_{\mathrm{c}}$ are the rate

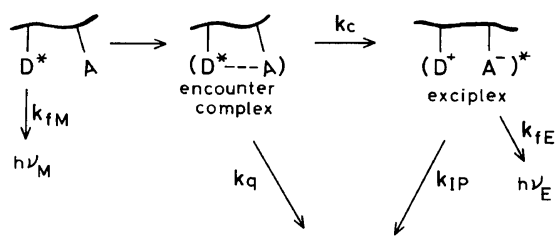

Scheme 1

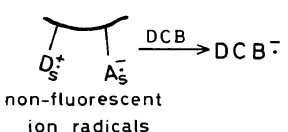

$$
\gamma=k_{c} /\left(k_{c}+k_{q}\right), \quad \Phi_{E}=\gamma k_{f E} \tau_{E}
$$

constants for the direct ion radical formation from the encounter complex, ion radical formation via the exciplex and exciplex formation, respectively. According to this scheme, the decrease of $\Phi_{\mathrm{E}}$ is greater than that of $\tau_{\mathrm{E}}$ by a factor of $\gamma(\gamma<1)$. 


\section{CONCLUSIONS}

The formation of the intramolecular exciplex in the vinyl copolymer system, its characteristics, and the electron-transfer process have been studied for the first time. As compared with the intermolecular exciplex of low-molecular-weight model system, the intramolecular exciplex of the vinyl copolymer system is characterized by ease of formation, the strong intensity of the fluorescence, and less sensitivity to the solvent polarity. As in the case of the intermolecular exciplex system, the formation of ion radicals in polar solvents has been shown also for the present intramolecular exciplex system of the copolymer, although ionic dissociation would be improbable for the copolymer system. However, charge separation has been attained by the addition of a stronger electron-acceptor to the copolymer solution. In that case, the electron-acceptor anion radical unit bound to the polymer chain acts as an electron supplier to the stronger electron-acceptor added, which contrasts in the case of the intermolecular exciplex system of low-molecular-weight compounds.

\section{REFERENCES}

1. H. Mikawa and M. Yokoyama, Kagaku, 30, 910 (1975).

2. Y. Nishijima, Koubunshi, 25, 830 (1976).

3. S. S. Yanari, F. A. Bovey, and R. Lumry, Nature, 200, 242 (1963); M. T. Vala, J. Haebig, and S. A. Rice, J. Chem. Phys., 43, 886 (1966); J. W. Longworth and F. A. Bovey, Biopolymers, 4, 1115 (1966); J.W. Longworth, Biopolymers, 4, 1131 (1966); Y. Nishijima, J. Polym. Sci., C, 31, 358 (1970), etc.
4. W. Klöpffer, J. Chem. Phys., 50, 2337 (1969); P. C. Johnson and H. W. Offen, J. Chem. Phys., 55, 2945 (1971); C. David, M. Piens, and G. Genskens, Eur. Polym. J., 8, 1291 (1972); W. Klöpffer and D. Fisher, J. Polym. Sci., C, 40, 43 (1973); K. Okamoto, A. Yano, S. Kusabayashi, and H. Mikawa, Bull. Chem. Soc. Jpn., 47, 749 (1974); M. Yokoyama, T. Tamamura, M. Atsumi, M. Yoshimura, Y. Shirota, and H. Mikawa, Macromolecules, 8, 107 (1975); G. E. Johnson, J. Chem. Phys., 62, 4697 (1975), etc.

5. L. A. Harrah, J. Chem. Phys., 56, 385 (1972); C. W. Frank and L. A. Harrah, J. Chem. Phys., 61, 1526 (1974), etc.

6. S. Tazuke and Y. Matsuyama, Macromolecules, 8, 280 (1975).

7. S. Tazuke, K. Sato, and F. Banba, Chem. Lett., 1321 (1975).

8. Y. Matsuyama and S. Tazuke, Polym. J., 8, 481 (1976).

9. S. Tazuke and Y. Matsuyama, Macromolecules, 10, 215 (1977).

10. S. Tazuke, K. Sato, and F. Banba, Macromolecules, 10, 1224.(1977).

11. H. Beens and A. Weller, Acta Physica Polon., 34, 593 (1968).

12. R. W. Strassburg, R. A. Gregg, and C. Walling, $J$. Am. Chem. Soc., 69, 2141 (1947).

13. M. Suzuki and M. Nagawa, J. Pharm. Soc. Jpn., 72, 305 (1952).

14. C. S. Marvel and C. G. Overberger, J. Am. Chem. Soc., 67, 2250 (1945).

15. C. G. Overberger and R. E. Allen, J. Am. Chem. Soc., 68, 722 (1946).

16. N. Yamamoto, Y. Nakao, and H. Tsubomura, Bull. Chem. Soc. Jpn., 39, 2603 (1966).

17. T. Okada, H. Oohari, and N. Mataga, Bull. Chem. Soc. Jpn., 43, 2750 (1970).

18. H. Knibbe, K. Rollig, F. P. Schafer, and A. Weller, J. Chem. Phys., 47, 1184 (1967). 